\title{
A semi-automated motion-tracking analysis of locomotion speed in the $C$. elegans transgenics overexpressing beta-amyloid in neurons
}

\author{
Kevin Machino ${ }^{1}$, Christopher D. Link ${ }^{2}$, Susan Wang ${ }^{1}$, Hana Murakami ${ }^{1}$ and Shin Murakami ${ }^{1 *}$ \\ ${ }^{1}$ Department of Basic Sciences, College of Osteopathic Medicine, Touro University California, Vallejo, CA, USA \\ 2 Institute of Behavioral Genetics, University of Colorado, Boulder, Boulder, CO, USA
}

Edited by:

Nektarios Tavernarakis, University of Crete, Foundation for Research and Technology-Hellas, Greece

\section{Reviewed by:}

Eirini Lionaki, Foundation for Research and Technology-Hellas, Greece

Vassiliki Nikoletopoulou,

Institute of Molecular Biology and Biotechnology, Greece

Maria Markaki, Foundation for Research and Technology-Hellas, Greece

\section{*Correspondence:}

Shin Murakami, College of Osteopathic Medicine, Touro University California, 1310 Club Drive, Mare Island, Vallejo, CA 94594, USA

e-mail:shin.murakami@tu.edu
Multi-Worm Tracker (MWT) is a real-time computer vision system that can simultaneously quantify motional patterns of multiple worms. MWT provides several behavioral parameters, including analysis of accurate real-time locomotion speed in the nematode, Caenorhabditis elegans. Here, we determined locomotion speed of the Alzheimer's disease $(A D)$ transgenic strain that over-expresses human beta-amyloid1-42 (A $\beta)$ in the neurons. The MWT analysis showed that the AD strain logged a slower average speed than the wild type (WT) worms. The results may be consistent with the observation that the $A D$ patients with dementia tend to show deficits in physical activities, including frequent falls. The AD strain showed reduced ability of the eggs to hatch and slowed hatching of the eggs. Thus, over-expression of $A \beta$ in neurons causes negative effects on locomotion and hatchability. This study sheds light on new examples of detrimental effects that $A \beta$ deposits can exhibit using $C$. elegans as a model system. The information gathered from this study indicates that the motion tracking analysis is a cost-effective, efficient way to assess the deficits of $A \beta$ over-expression in the $C$. elegans system.

Keywords: Alzheimer's disease, beta amyloid, age-related memory impairment (AMI), frailty, behavioral aging, motion tracking, automated system, Alzheimer's disease in children

\section{INTRODUCTION}

Alzheimer's disease $(\mathrm{AD})$ is a type of amyloidosis and is the major neurodegenerative disorder that causes dementia. Amyloidosis is characterized by fibril deposits that contain at least 30 fibril proteins in humans and 10 fibril proteins in animals, according to the 2012 the Nomenclature Committee of the International Society of Amyloidosis (Sipe et al., 2012). Of them, accumulation of $A \beta$ has been observed in the $\mathrm{AD}$ and congophilic cerebral angiopathy (CAA) (Selkoe, 2001; Revesz et al., 2003). AD leads to cognitive deficits due to abnormal deposits of $\beta$-amyloid $(A \beta)$ peptides in the brain, while CAA is characterized by vascular deposits in the central nervous system, in which hemorrhage is a major clinical feature (Samarasekera et al., 2012). CAA is often observed in $\mathrm{AD}$ patients, in which case it has been diagnosed as $\mathrm{AD}$ (Wright, 2013).

In $\mathrm{AD}$, deposits of the $\mathrm{A} \beta$ peptides arise from the proteolytic processing of amyloid precursor proteins (APP), commonly observed in patients with $\mathrm{AD}$ (Nicholson et al., 2012). $\mathrm{A} \beta$ deposits and tau tangles are well known hallmarks for $\mathrm{AD}$, which may trigger inflammation worsening the disease (Nicholson et al., 2012; Jack and Holtzman, 2013). A $\beta$ toxicity may be linked to tau hyperphysphorylation observed in tau tangles (Lloret et al., 2011; Ermak and Davies, 2013). Although it is believed that $A \beta$ is involved in initiation of $\mathrm{AD}$ in the presence of tau pathologies and that tau is correlated with severity of $\mathrm{AD}$ (reviewed in LaFerla, 2010; Castillo-Carranza et al., 2014), molecular mechanisms of
$\mathrm{AD}$ remain unclear. Details of clinical pathogenesis and biomarkers have been reviewed elsewhere (Jack and Holtzman, 2013).

This study aimed to assess the effects of $A \beta$ toxicity on a behavioral parameter, average speed of movement. A transgenic strain overexpressing the signal peptide:: $\mathrm{A} \beta^{1-42}$ in the neurons (Dosanjh et al., 2010; Lublin and Link, 2013) was used for this study. Over-expression of $A \beta$ in the neurons may affect the serotonin system (Dosanjh et al., 2010). Previously, locomotion has been assessed in a manual assay that measures the rate of body bend, which is an indirect measure with relatively high variability (Dosanjh et al., 2010; Lublin and Link, 2013); the manual assay may have been missed early signs of motility defect. In addition, another neural $A \beta$ strain is tagged by a behavioral marker (i.e., roller phenotype) (McColl et al., 2012), which is difficult to assess locomotion speed. We used behavioral tracking software, Multi Worm Tracker (MWT) (Swierczek et al., 2010), to record and collect data on the speed. MWT is a program designed to quantify the behavior of multiple worms on a petri plate with minimal human effort. The main advantage is that it allows for a more accurate and detailed assessment of locomotion speed compared to the traditional method that measures body bends. Thus, our hypothesis is that the strain overexpressing $A \beta$ in the neurons show defects in locomotion speed. We reason that motion tracking analysis, including MWT analysis, should contribute to understanding the harmful effects of $A \beta$ toxicity in C. elegans. 


\section{MATERIALS AND METHODS STRAINS, MEDIA, AND STAINING}

Wild-type strain, N2, was used (referred to as WT strain). As a control, we also used the $s m g-1^{t s}$ strain $\left[s m g-1^{t s}(c c 546)\right]$, which generated similar results as N2 in the assays (i.e., locomotion and hatchability) used in this study (data not shown). The strain CL2355 [smg- $1^{\text {ts }}(c c 546) ; s n b-1:: A \beta_{1-42}::$ long $3^{\prime}$-UTR] that utilizes the $C$. elegans promotor of the synaptobrevin ( $s n b$ 1) gene to cause a pan-neuronal overexpression of the signal peptide::A $\beta^{1-42}$ (Dosanjh et al., 2010; Lublin and Link, 2013) was used (referred to as $\mathrm{AD}$ strain). For simplicity, the transgenic strain CL2355 is referred to as the $\mathrm{AD}$ strain for the duration of the paper. All strains were maintained at $15^{\circ} \mathrm{C}$ in a nematode growth media (NGM) spotted with Escherichia coli, OP50, as a food source (Murakami et al., 2005). The following procedure adopted from Stiernagle (2006) was used to prepare NGM plates. Before autoclaving, $17 \mathrm{~g}$ agar, $2.5 \mathrm{~g}$ peptone, $3 \mathrm{~g} \mathrm{NaCl}$, and $975 \mathrm{~mL}$ distilled $\mathrm{H}_{2} \mathrm{O}$ was added to a flask and covered with aluminum foil. Then the flask was autoclaved for $50 \mathrm{~min}$. After autoclaving the mixture, $1 \mathrm{~mL}$ of $1 \mathrm{M}$ $\mathrm{CaCl}_{2}, 1 \mathrm{~mL}$ of $5 \mathrm{mg} / \mathrm{mL}$ cholesterol in ethanol, $1 \mathrm{~mL}$ of $1 \mathrm{M}$ $\mathrm{MgSO}_{4}$, and $25 \mathrm{~mL}$ of $1 \mathrm{M} \mathrm{KPO}_{4}(\mathrm{pH} 6.0$ ) was added to the flask. Then petri plates were filled $2 / 3$ with agar. Plates were left at room temperature for 2-3 days to allow excess moisture to evaporate. For immunofluorescence staining, we used the procedure described in Link (1995). Transgenic worms were fixed, permeabilized, and stained with the anti-A $\beta$ monoclonal antibody 4G8 and anti-TOR-2 polyclonal sera as a counterstain. DNA was visualized using 4',6-diamidino-2-phenylindole (DAPI).

\section{GROWTH CONDITIONS}

Strains were grown on NGM agar plates at $15^{\circ} \mathrm{C}$. To assess hatchability, eggs were layed on NGM plates at $15^{\circ} \mathrm{C}$ and incubated at the temperature indicated in the text $\left(15\right.$ or $\left.25^{\circ} \mathrm{C}\right)$. Unhatched eggs were counted after $24 \mathrm{~h}$ or the time specified in the text. Hatched eggs (larvae) were also counted to confirm the result. To prepare adult worms, eggs were layed and grown into adults for 4 days. Adults at the age of day 5 (i.e., 1 day after they start to lay eggs) was defined as "younger adults." Adults at the age of day 7 was defined as "middle-aged adults." Adults at the age of day 13-14 was defined as "older adults."

\section{MULTI WORM TRACKER (MWT) ANALYSIS}

Videos of the worms were recorded under a stereomicroscope using ToupView, a video capturing software (Amscope.com, Irvine, CA). The parameters of $3 \mathrm{~min}$ for duration and $6 \mathrm{~s}$ for bin size was set when capturing the video, which created a video file that was $35 \mathrm{~s}$ in length. To analyze the speed of the worms, the video file was uploaded into MWT, a behavioral tracking software (Swierczek et al., 2010). In a typical assay, about five worms in a microscopic field was computed and average speed of the worms were calculated. A measure for speed (in pixels/s) was made at a series of ages indicated in the text. We used the conversion rate in the system: $1 \mathrm{pixel} / \mathrm{s}=0.035 \mathrm{~cm} / \mathrm{s}$ at the images of 72 PPI (pixels per inch). Death was determined by observing no movement of the worms. Statistical analysis has been performed by ANOVA using NCSS 2007 statistics software (NCSS, LLC, Kaysville, Utah, USA).

\section{RESULTS}

We sought to assay the locomotion of the transgenic C. elegans strain CL2355 (Dosanjh et al., 2010), which expresses a human $\mathrm{A} \beta \beta_{42}$ minigene under the control of the pan-neuronal synaptobrevin $(s n b-1)$ promoter. Expression of $\mathrm{A} \beta$ using this promoter leads to intraneuronal deposition of $A \beta$, particularly detectable in the nerve ring area (Figure 1).

Video images of the worms on NGM agar were captured for $3 \mathrm{~min}$ and the speed of the worms was determined using MWT. We compared the results of the WT (wild type) and AD strains (over-expressing amyloid beta) (Materials and Methods). As described in the Method, adults that had grown for 5 days were defined as "younger adults." Adults that had grown for 7 days were defined as "middle-aged adults." Adults that had grown for 13 days were defined as "older adults."

\section{THE AD TRANSGENIC STRAIN SHOWING SLOWER MOVEMENT}

Table 1 summarizes the results. For the younger adults (day 5), WT had a mean speed of $0.18 \pm 0.15 \mathrm{~cm} / \mathrm{s}$ whereas the AD had a mean speed of $0.05 \pm 0.06 \mathrm{~cm} / \mathrm{s}(p>0.0001$; Figure 2A;

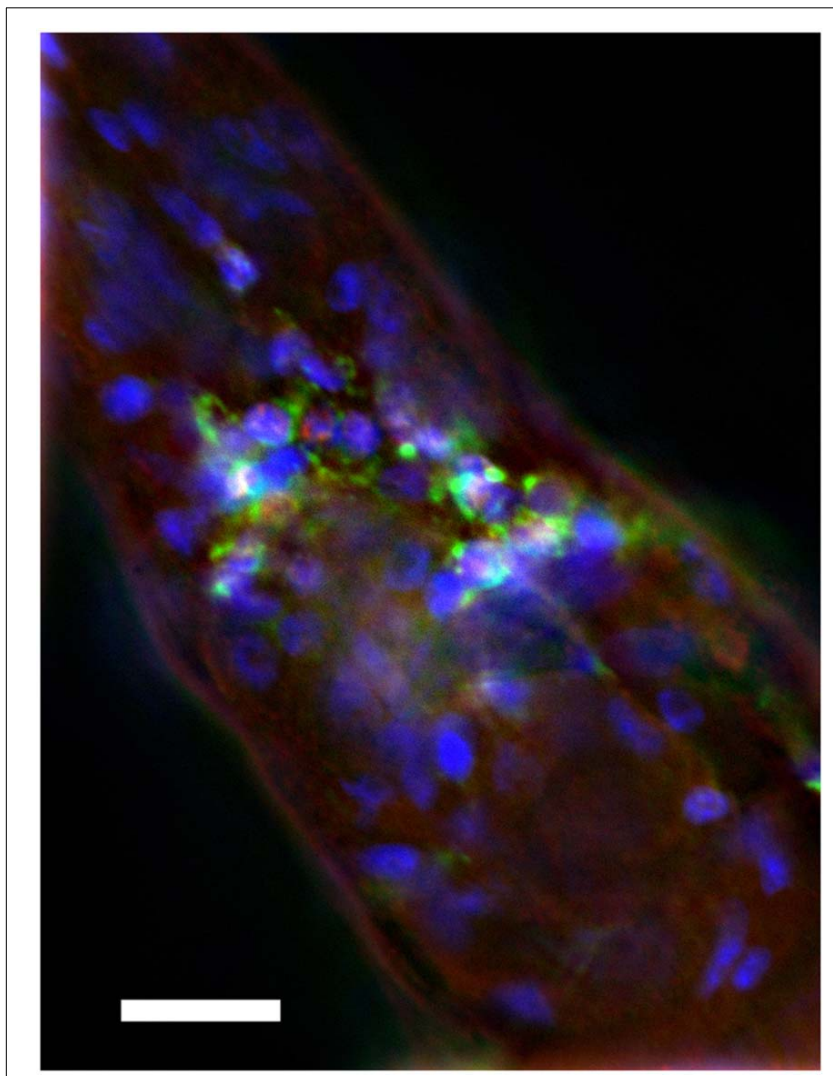

FIGURE 1 | Intraneuronal deposition of A $\beta$ in transgenic $\boldsymbol{C}$. elegans. Anterior region of transgenic $C$. elegans is shown. The strain was immune-stained by anti-A $\beta$ monoclonal antibody $4 \mathrm{G} 8$ (green), anti-TOR-2 polyclonal sera (red), and DAPI (blue). Note cytoplasmic accumulation of $A \beta$ in nerve ring neurons. Size bar $=10 \mu \mathrm{M}$ 
Table 1 | Locomotion speed (mean \pm standard deviation) as assessed by MWT analysis for various stages in the life cycle $(* p<0.0001)$.

\begin{tabular}{lcc}
\hline Life stage (day \#) & Type of strain & Average speed $\mathbf{( c m} / \mathbf{s})$ \\
\hline Younger adult (day 5) & WT & $0.18 \pm 0.15$ \\
& AD & $0.05 \pm 0.06^{*}$ \\
\hline (day 6) & WT & $0.52 \pm 0.25$ \\
& AD & $0.21 \pm 0.17^{*}$ \\
\hline Middle-aged (day 7) & WT & $0.43 \pm 0.19$ \\
& AD & $0.05 \pm 0.05^{*}$ \\
\hline (day 10) & WT & $0.10 \pm 0.11$ \\
& AD & $0.08 \pm 0.12$ \\
\hline Older adult (day 13) & WT & $0.11 \pm 0.07$ \\
& AD & $0.12 \pm 0.07$ \\
\hline
\end{tabular}

Table 1). Slow locomotion speed in the AD strain compared to WT was observed from day 5 (younger adults) to day 7 (middle age) ( $p>0.0001$; Figures 2A,B; Table 1). Interestingly, there was a peak of locomotion speed at the age of day 5 (Figure 2E), which was consistent with previous study as assessed in a classical assay that measured body bends, an indicator of locomotion speed (Murakami et al., 2008).

Slow locomotion speed in the AD strain was also evident in the still images (Figure 3) and Supplementary Video (Supplementary Figure). In the sill images taken every $7 \mathrm{~s}$ (Figure 2), the WT worms were located in different positions (Figure 3A), while the AD worms were nearly stagnant from 0 to $35 \mathrm{~s}$ (Figure 3B). Thus, a difference in the worm movements was clearly visible.

Older worms at day 10 and 13 showed similar results for the WT and AD strains. Day $10 \mathrm{WT}$ worms had an average speed of $0.10 \pm 0.11 \mathrm{~cm} / \mathrm{s}$ whereas Day $10 \mathrm{AD}$ worms moved at an average speed of $0.08 \pm 0.12 \mathrm{~cm} / \mathrm{s}$ for four worms (Figure 2C; Table 1). Finally, in the older adult at the age of Day 13, WT worms logged an average speed of $0.11 \pm 0.07 \mathrm{~cm} / \mathrm{s}$ and Day $13 \mathrm{AD}$ worms showed similar locomotion speed $(0.12 \pm 0.07 \mathrm{~cm} / \mathrm{s})$ (Figure 2D; Table 1). Overall, worms with overexpressed $A \beta$ resulted in slower movement up to middle-aged (Figure 2).

\section{A $\beta$ TOXICITY IN THE EMBRYONIC STAGE}

Hatching of the eggs from the WT (N2) and AD (CL2355) strains were compared. We measured total number of unhatched eggs $24 \mathrm{~h}$ at restrictive temperature $\left(25^{\circ} \mathrm{C}\right)$ after egg lay. We also counted hatched larvae to confirm the results. In the WT strain, there were $16.3 \%(37 / 227)$ eggs that remained unhatched and 83.7\% (190/227) hatched (Figure 4A). For the AD strain, 89.2\% $(182 / 204)$ eggs remained unhatched and 10.8\% (22/204) hatched. The rate of hatching in the control strain $\left(s m g-1^{t s}\right)$, was similar to WT (data now shown), excluding the possibility that the background mutation lowered the rate of hatching. Thus, the AD strain showed an approximately 7.8 folds lower hatching after $24 \mathrm{~h}$ of egg lay. We also investigated the time course of hatchability at permissive temperature, $15^{\circ} \mathrm{C}$. Most of the eggs in the WT strain hatched 1 day after egg lay (Day 2; Figure 4B). In contrast, the AD strain hatched much slower than the WT strain, taking 2 days after egg lay (Day 3; Figure 4B). Thus, the AD strain shows reduced hatching and a delayed timing of egg hatching. We also observed a low rate of fecundity (Blood size: WT, $248 \pm 22, n=10 ; \mathrm{AD}$, $50 \pm 12, n=20 ; p<0.001)$.

\section{DISCUSSION}

In this study, we used MWT analysis to measure locomotion speed. Compared to a manual assay to measure the number of body bends, MWT analysis provides a more accurate measure of multiple worms for a longer time (MWT for $3 \mathrm{~min}$; Manual assay for $1 \mathrm{~min}$ ). Young wild-type worm (locomotion speed: $0.18 \pm$ $0.15 \mathrm{~cm} / \mathrm{s}$ ) was within the range of previously reported results (lower range of $0.13 \mathrm{~cm} / \mathrm{s}$, Ramot et al., 2008; upper range of $0.22 \mathrm{~cm} / \mathrm{s}$, Ryu and Samuel, 2002). They have also reported locomotion speed, ranging from 0.14 to $0.33 \mathrm{~cm} / \mathrm{s}$ at the temperatures ranging from 17 to $27^{\circ} \mathrm{C}$. The groups used other worm tracking systems, which suggests that MWT analysis results are consistent with those from the other worm tracking systems. It is worth noting that our MWT system is one of the most cost effective systems. The total cost was estimated to be up to US $\$ 200$ for a routine USB-camera (as of April, 2014; our cost was zero since we recycled the camera, excluding the cost of software, computer, and microscope), which is much less than the cost estimated for MWT elsewhere (US\$7,000) (Husson et al., 2012).

The AD strain showed an approximately 7.8-fold decrease in hatching compared to the WT strain. Likewise, the AD strain showed reduced hatching even at the permissive temperature, where $A \beta$ is expressed, but at a lower level. The reduced level of hatching seen in the $\mathrm{AD}$ strain could be attributed to $\mathrm{A} \beta$ overexpression, suggesting the negative effect of $A \beta$ on embryogenesis due to its toxicity. It is worth noting that some CL2355 worms are completely sterile (no eggs laid). This is a surprising observation if the defects in the CL2355 strain are restricted to neurons, as fertility in C. elegans is not strongly neuronally regulated. The fertility/sterility phenotypes of CL2355 were observed in the precursor extrachromosomal line, suggesting that these defects are not due to gene disruption caused by chromosomal integration of the transgene. We also observed that worms show larval arrest and death, suggesting defects in development caused by $\mathrm{A} \beta$ (data not shown).

The findings also suggest that $A \beta$ accumulation has detrimental effects on locomotion speed in the adult life cycle, except for late-life in which all worms move poorly. In younger adults and middle-aged adults (corresponding to Day 5 and 7, respectively), the AD worms exhibited a slower speed than the WT worms (Figure 1). The middle-aged $\mathrm{AD}$ worms showed the greatest amount of difference from that of the WT worms; the approximate 8.3-fold difference in speed indicates that the presence of $\mathrm{A} \beta$ is correlated with the slower movement in the worms. This may be consistent with frailty seen in the Alzheimer's patients (Koch et al., 2013; Kulmala et al., 2014) and gait problems in the mouse models of AD (APP and APP/PS1 mice) (Lalonde et al., 2012; Wang et al., 2012a), though some other mice models [APP23, J20, APP + PDAPP, PS1 [Tg2576 + PS1 (M146L)], TgCRND8, TG2576, and $3 \times$ Tg-AD mice] show increased locomotion due to 


\section{A}

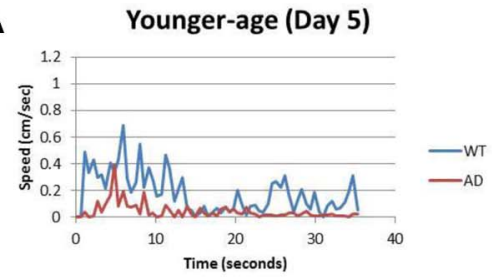

B

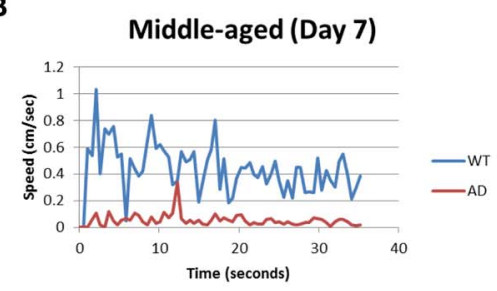

C

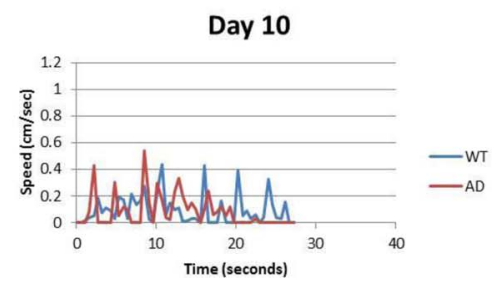

FIGURE 2 | The AD strain exhibits slower speeds than WT in the life cycle. (A) The graph represents the locomotion speed of adult worms measured at the age of day 5 (younger age). (B) The graph represents the speed for worms measured at the age of day 7 (middle age). (C) The graph represents the speed for middle-aged worms measured at the age of day 10 .
D

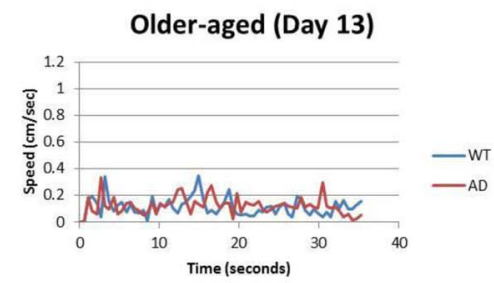

E

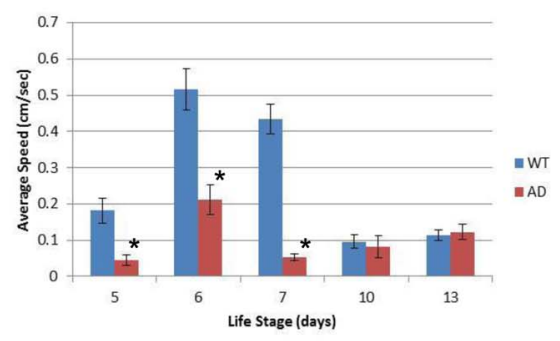

(D) The graph represents the speed for older adult worms measured the age of day 13 (older age). (E) Comparison of the average speeds for WT (blue) and $A D$ (red) worms at various ages over the course of their life span. Error bars indicate standard error of the mean. ${ }^{*} p>0.0001$. WT worms (blue); and AD worms (red). See also Table 1.

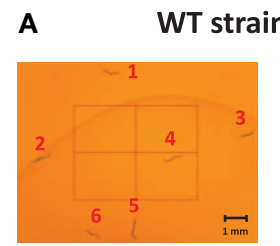

$0 \mathrm{sec}$

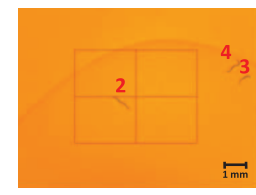

$14 \mathrm{sec}$

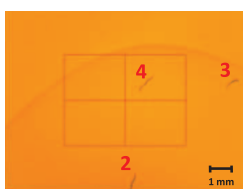

$28 \mathrm{sec}$

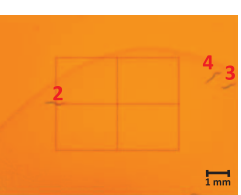

$7 \mathrm{sec}$

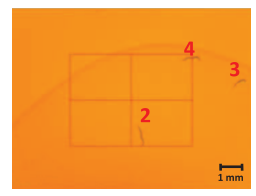

$21 \mathrm{sec}$

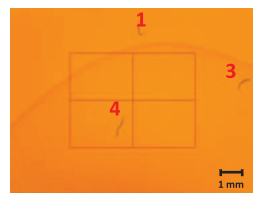

$35 \mathrm{sec}$

FIGURE 3 | Images of middle-aged (day 7) worms on the NGM plates at various time points. The images captured were taken every $7 \mathrm{~s}$ from 0 to $35 \mathrm{~s}$. The worms were labeled 1-6 for the WT and 1-5 for the $A D$ strain so that the difference in movement

\section{B}

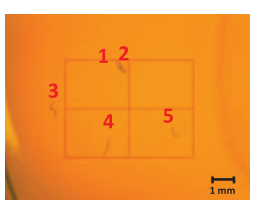

$0 \mathrm{sec}$

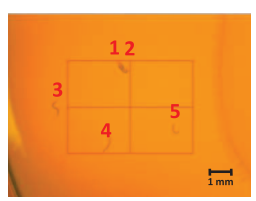

$14 \mathrm{sec}$

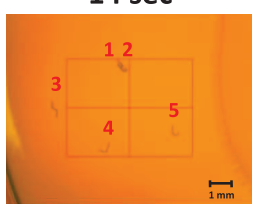

$28 \mathrm{sec}$

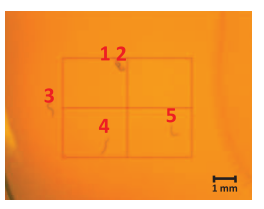

$7 \mathrm{sec}$

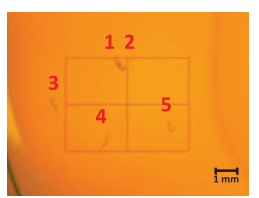

$21 \mathrm{sec}$

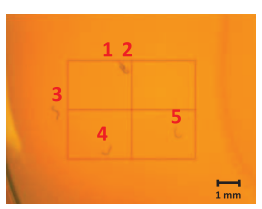

$35 \mathrm{sec}$

could be seen between and amongst frames. (A) An example of the WT worms. They moved throughout the plate as time progressed. (B) An example of the $A D$ worms. They hardly moved as time elapsed. 


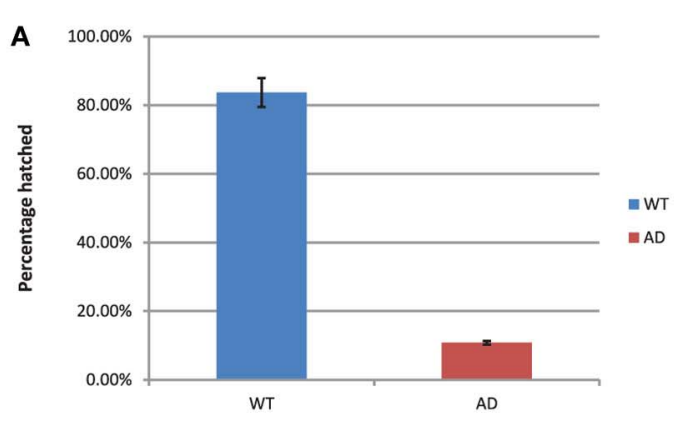

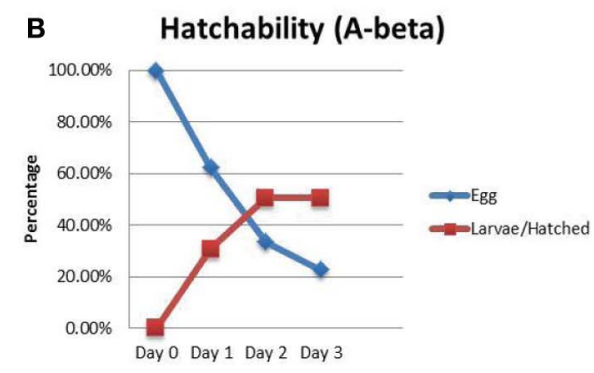

FIGURE 4 | Rate of egg hatching (hatchability) in the WT and AD strains. (A) Percentage of eggs unhatched after $24 \mathrm{~h}$. There were more $A D$ eggs (red) that remained unhatched compared to the WT eggs (blue).

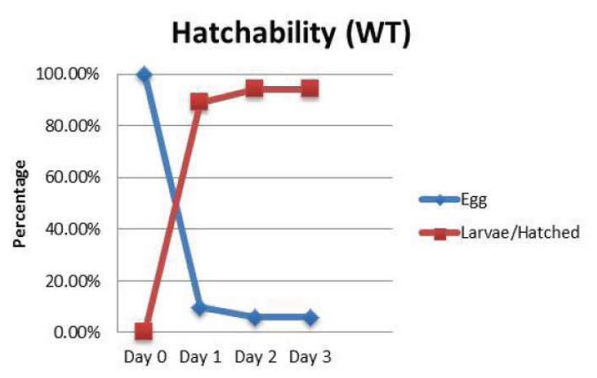

(B) Time course of the percentage of eggs that hatched from day 0 (the day of egg lay) to day 3 for both strains. Error bars indicate standard error of the mean. aggression and other behavioral problems (Webster et al., 2014). Thus, locomotion is not always defective in $\mathrm{AD}$ patients and in $\mathrm{AD}$ models but rather altered. It is critical to assess locomotion in each $\mathrm{AD}$ system. In addition to our finding, locomotion defects have been observed in the fruit fly model of $A \beta$ toxicity, while paralysis has been observed in the other strains over-expressing $\mathrm{A} \beta$ in the muscles and in the neurons (McColl et al., 2012; Wang et al., 2012b; Lublin and Link, 2013; Prüßing et al., 2013; CarrilloMora et al., 2014). Technical difficulties in the previous studies have been discussed above.

This study provides evidence that $A \beta$ toxicity affects the embryonic stage as well as the adult phases. Since the AD transgenic strain used in this study has $A \beta$ overexpression, the data can be used to assess the effects of $A \beta$ toxicity on embryonic and behavioral parameters. Assessing the hatchability and speed of the worms allows for the analysis of the strains and the impact $A \beta$ toxicity has on embryonic health and movement. The research will be beneficial as $\mathrm{AD}$ is one of the most common causes of dementia, and the number of Americans aged 65 and older affected by $\mathrm{AD}$ is predicted to triple by 2050 (Hebert et al., 2013). Currently, there are drugs there are drugs that can treat memory impairment in $\mathrm{AD}$ patients, but there are no cure for the disease itself. It is worth noting that a chaperone, HSP-16, is strongly associated with the $A \beta$ deposits in the neuronal expression lines (reviewed in Lublin and Link, 2013). The future direction of this study aims to explore the relationship between $\mathrm{AD}$ drugs and potential $\mathrm{A} \beta$ clearance. The hope is that future studies will demonstrate the effectiveness of current FDA-approved $\mathrm{AD}$ treatment interventions to help alleviate the $\mathrm{A} \beta$ buildup seen in $\mathrm{AD}$ patients and enhance future treatments by potentially slowing or stopping the disease.

\section{ACKNOWLEDGMENTS}

We thank Dr. Rex Kirr (Janeila Farm Research Campus, Ashburn, VA) for technical assistance of MWT analysis and Mr. Chris Koebler for editing the manuscript. The work was supported by research support from the Tour University California (Shin Murakami) and NIH grant R01 AG012423 (Christopher D. Link).

\section{SUPPLEMENTARY MATERIAL}

The Supplementary Material for this article can be found online at: http://www.frontiersin.org/journal/10.3389/fgene. 2014.00202/abstract

Supplementary Video | Video images of day $\mathbf{7}$ worms shown in Figure 2.

(Video 1). Video shows the WT strain (File name: Machino et al. WT strain Day 7.mwv). (Video 2) Video shows the AD strain (File name: Machino et al. AD strain Day 7.mwv). As described in Materials and Methods, 3 min-long videos were shortened into $35 \mathrm{~s}$ in length. Due to the conversion from AVI to MP4 format to reduce the size of the files, the video have lower quality compared to the originals.

\section{REFERENCES}

Carrillo-Mora, P., Luna, R., and Colín-Barenque, L. (2014). Amyloid beta: multiple mechanisms of toxicity and only some protective effects? Oxid. Med. Cell. Longev. 2014:795375. doi: 10.1155/2014/795375

Castillo-Carranza, D. L., Guerrero-Muñoz, M. J., and Kayed, R. (2014). Immunotherapy for the treatment of Alzheimer's disease: amyloid- $\beta$ or tau, which is the right target? ImmunoTargets and Therapy 3, 19-28. doi: 10.2147/ ITT.S40131

Dosanjh, L. E., Brown, M. K., Rao, G., Link, C. D., and Luo, Y. (2010). Behavioral phenotyping of a transgenic Caenorhabditis elegans expressing neuronal amyloid-beta. J. Alzheimers. Dis. 19, 681-690. doi: 10.3233/JAD2010-1267 
Ermak, G., and Davies, K. J. (2013). Chronic high levels of the RCAN1-1 protein may promote neurodegeneration and Alzheimer disease. Free Radic. Biol. Med. 62, 47-51. doi: 10.1016/j.freeradbiomed.2013.01.016

Hebert, L. E., Weuve, J., Scherr, P. A., and Evans, D. A. (2013). Alzheimer disease in the United States (2010-2050) estimated using the 2010 census. Neurology 80, 1778-1783. doi: 10.1212/WNL.0b013e31828726f5

Husson, S. J., Costa, W. S., Schmitt, C., and Gottschalk, A. (2012). Keeping Track of Worm Trackers. WormBook, the C. elegans Research Community. Availbale online at: http://www.wormbook.org

Jack, C. R. Jr., and Holtzman, D. M. (2013). Biomarker modeling of Alzheimer's disease. Neuron. 80, 1347-1358. doi: 10.1016/j.neuron.2013.12.003

Koch, G., Belli, L., Giudice, T. L., Lorenzo, F. D., Sancesario, G. M., Sorge, R., et al. (2013). Frailty among Alzheimer's disease patients. CNS Neurol. Disord. Drug Targets. 12, 507-511. doi: 10.2174/1871527311312040010

Kulmala, J., Nykänen, I., Mänty, M., and Hartikainen, S. (2014). Association between Frailty and Dementia: a population-based study. Gerontology. 60, 16-21. doi: 10.1159/000353859

LaFerla, F. M. (2010). Pathways linking Abeta and tau pathologies. Biochem. Soc. Trans. 38, 993-995. doi: 10.1042/BST0380993

Lalonde, R., Fukuchi, K., and Strazielle, C. (2012). Neurologic and motor dysfunctions in APP transgenic mice. Rev. Neurosci. 23, 363-379. doi: 10.1515/revneuro-2012-0041

Link, C. D. (1995). Expression of human beta-amyloid peptide in transgenic Caenorhabditis elegans. Proc. Natl. Acad. Sci. U.S.A. 92, 9368-9372.

Lloret, A., Badia, M. C., Giraldo, E., Ermak, G., Alonso, M. D., Pallardó, F. V., et al. (2011). Amyloid- $\beta$ toxicity and tau hyperphosphorylation are linked via RCAN1 in Alzheimer's disease. J. Alzheimers. Dis. 27, 701-709. doi: 10.3233/JAD-2011110890

Lublin, A. L., and Link, C. D. (2013). Alzheimer's disease drug discovery: in vivo screening using Caenorhabditis elegans as a model for $\beta$-amyloid peptide-induced toxicity. Drug Discov. Today Technol. 10, e115-e119. doi: 10.1016/j.ddtec.2012.02.002

McColl, G., Roberts, B. R., Pukala, T. L., Kenche, V. B., Roberts, C. M., Link, C. D., et al. (2012). Utility of an improved model of amyloid-beta $\left(A \beta_{1-42}\right)$ toxicity in Caenorhabditis elegans for drug screening for Alzheimer's disease. Mol. Neurodegener. 7:57. doi: 10.1186/1750-1326-7-57

Murakami, H., Bessinger, K., Hellmann, J., Luerman, G. C., and Murakami, S. (2008). Manipulation of serotonin signal suppresses early phase of behavioral aging in Caenorhabditis elegans. Neurobiol. Aging 29, 1093-100. doi: 10.1016/j.neurobiolaging.2007.01.013

Murakami, H., Bessinger, K., Hellmann, J., and Murakami, S. (2005). Agingdependent and independent regulation of learning by insulin/IGF-1 signal in C. elegans. J. Neurosci. 25, 10894-10904. doi: 10.1523/JNEUROSCI.360004.2005

Nicholson, A. M., Wold, L. A., Walsh, D. M., and Ferreira, A. (2012). $\beta$-Amyloid carrying the dutch mutation has diverse effects on calpain-mediated toxicity in hippocampal neurons. Mol. Med. 18, 178-185. doi: 10.2119/molmed.2011. 00366

Prüßing, K., Voigt, A., and Schulz, J. B. (2013). Drosophila melanogaster as a model organism for Alzheimer's disease. Mol. Neurodegener. 8:35 doi: 10.1186/17501326-8-35

Ramot, D., MacInnis, B. L., Lee, H. C., and Goodman, M. B. (2008). Thermotaxis is a robust mechanism for thermoregulation in Caenorhabditis elegans nematodes. J. Neurosci. 1928, 12546-12557. doi: 10.1523/JNEUROSCI.285708.2008
Revesz, T., Ghiso, J., Lashley, T., Plant, G., Rostagno, A., Frangione, B., et al. (2003). Cerebral amyloid angiopathies: a pathologic, biochemical, and genetic view. J. Neuropathol. Exp. Neurol. 62, 885-898.

Ryu, W. S., and Samuel, A. D. (2002). Thermotaxis in Caenorhabditis elegans analyzed by measuring responses to defined Thermal stimuli. J. Neurosci. 22, 5727-5733.

Samarasekera, N., Smith, C., and Al-Shahi Salman, R. (2012). The association between cerebral amyloid angiopathy and intracerebral haemorrhage: systematic review and meta-analysis. J. Neurol. Neurosurg. Psychiatry 83, 275-281. doi: 10.1136/jnnp-2011-300371

Selkoe, D. J. (2001). Alzheimer's disease: genes, proteins, and therapy. Physiol. Rev. $81,741-766$.

Sipe, J. D., Benson, M. D., Buxbaum, J. N., Ikeda, S., Merlini, G., Saraiva, M. J., et al. (2012). Nomenclature Committee of the International Society of Amyloidosis. Amyloid fibril protein nomenclature: 2012 recommendations from the Nomenclature Committee of the International Society of Amyloidosis. Amyloid 19, 167-170. doi: 10.3109/13506129.2012.734345

Stiernagle, T. (2006). "Maintenance of C. elegans," in WormBook, Vol. 11, 1-11. doi: 10.1895/wormbook.1.101.1

Swierczek, N. A., Giles, A. C., Rankin, C. H., and Kerr, R. A. (2010). Highthroughput behavioral analysis in C. elegans. Nat Methods 8, 592-598. doi: 10.1038/nmeth.1625

Wang, H., He, J., Zhang, R., Zhu, S., Wang, J., Kong, L., et al. (2012a). Sensorimotor gating and memory deficits in an APP/PS1 double transgenic mouse model of Alzheimer's disease. Behav. Brain Res. 233, 237-243. doi: 10.1016/j.bbr.2012.05.007

Wang, L., Chiang, H.-C., Wu, W., Liang, B., Xie, Z., Yao, X., et al. (2012b). Epidermal growth factor receptor is a preferred target for treating Amyloid$\beta$-induced memory loss Proc. Natl. Acad. Sci. U.S.A. 109, 16743-16748. doi: 10.1073/pnas.1208011109

Webster, S. J., Bachstetter, A. D., Nelson, P. T., Schmitt, F. A., and Van Eldik, L. J. (2014). Using mice to model Alzheimer's dementia: an overview of the clinical disease and the preclinical behavioral changes in 10 mouse models. Front. Genet. 5:88. doi: 10.3389/fgene.2014.00088

Wright, C. B. (2013). "Etiology, clinical manifestations, and diagnosis of vascular dementia," in UpToDate, eds S. T. DeKosky and A. F. Eichler (Waltham, MA: UpToDate).

Conflict of Interest Statement: The authors declare that the research was conducted in the absence of any commercial or financial relationships that could be construed as a potential conflict of interest.

Received: 06 May 2014; accepted: 17 June 2014; published online: 04 July 2014. Citation: Machino K, Link CD, Wang S, Murakami H and Murakami S (2014) A semi-automated motion-tracking analysis of locomotion speed in the C. elegans transgenics overexpressing beta-amyloid in neurons. Front. Genet. 5:202. doi: 10.3389/ fgene.2014.00202

This article was submitted to Genetics of Aging, a section of the journal Frontiers in Genetics.

Copyright (c) 2014 Machino, Link, Wang, Murakami and Murakami. This is an openaccess article distributed under the terms of the Creative Commons Attribution License (CC BY). The use, distribution or reproduction in other forums is permitted, provided the original author(s) or licensor are credited and that the original publication in this journal is cited, in accordance with accepted academic practice. No use, distribution or reproduction is permitted which does not comply with these terms. 